\title{
Field Evaluation of New Ovicidal Acaricide (Tafethion) against Two Major Insect Pests of Apple in Kashmir, India
}

\author{
Asma Sherwani*, Malik Mukhtar Ahmad, Muneer Ahmad Sofi and Saima Maqsood
}

Division of Entomology, Sher-e-Kashmir University of Agricultural Sciences and Technology, Shalimar Srinagar, Kashmir 190025 J \& K, India

*Corresponding author

\section{A B S T R A C T}

Evaluation of newer acaricide Tafethion against San Jose scale Quadraspidiotus perniciosus Comstock and European red mite Panonychus ulmi Koch was evaluated for bio efficacy, phytotoxicity and effect on natural enemies in apple orchards at two locations

\begin{tabular}{|l|}
\hline Key w or d s \\
Quadraspidiotus \\
perniciosus, \\
Malus domestica. \\
\hline Article Info \\
\hline Accepted: \\
29 June 2017 \\
Available Online: \\
10 July 2017 \\
\hline \hline
\end{tabular}
in Kashmir during the year 2014 and 2015. Ethion was used as standard check for conducting the experiment. Both the acaricides were sprayed during dormant and summer seasons to suppress different stages of the pests. The acaricides were sprayed with the concentrations viz., $1.25,1.0$ and $0.75 \%$ in both the seasons. All the concentrations significantly reduced the insect pest populations over the standard check. Highest percent mortality of 68.21 and 77.41 were recorded against San Jose scale and 77.13 and 82.49 against European red mite at a concentration of $1.25 \%$ during dormant and summer seasons respectively. None of the phytotoxicity effects were seen on the apple trees. Amblyseius, Stethorus punctum and Coccinellids were the key predators found feeding on European red mite and San Jose scale respectively. Maximum cumulative mean mortality of natural enemies was found to be $36.79 \%$ at $1.25 \%$ concentration of Tafethion. The findings of the study revealed that spraying of the acaricide results in considerable reduction in the rate of infestation of European red mite and San Jose scale on apple trees at both the locations.

\section{Introduction}

Apple Malus domestica (Borkh) is remarkably predominant among all other temperate fruits in Jammu and Kashmir. However, the production and quality of apple is affected by a number of serious pests causing heavy economic loss to the crop. The major insect pests attacking this crop are San Jose scale, European Red Mite, Two spotted mite and Apple wooly aphid. Among all these pests San Jose scale and European red mite has established itself as a serious pest of apple trees in Jammu and Kashmir (Anonumous, 2014; Bhalla and Gupta, 1993). Since these pests have been accidentally introduced to many countries and are considered major pests in most regions of the world where deciduous fruits are grown (Madson and Morgan, 1970). The damage by San Jose scale Quadraspidiotus perniciosus (Comstock) is caused by nymphs and female scales, which suck the sap from branches, twigs and fruits, weakens the plant and leaves, render the fruit unacceptable and unmarketable. European Red Mite, Panonychus ulmi (Koch) is another serious pest feeding on foliage and thereby reducing 
the yield of the plant. Keeping in view the severity of the pests the experiment was undertaken to test the bio efficacy of Tafethion, its phytotoxicity, impact on quality of apple yield and effect on natural enemies in the apple ecosystem of Kashmir Valley.

\section{Materials and Methods}

To evaluate the effectiveness of Tafethion against San Jose scale and European red mite on apple, field trial was carried out in the farmer holding apple orchard at Wakura and Zaznah locations of Ganderbal district of Kashmir during the year 2014 and 2015. The orchard had apple trees of Red Delicious cultivar having uniform size and age of 15 years. The orchard was selected taking into account the outbreak and damage caused by the insect pests. The experiment was laid in a randomized block design (RBD) with seven treatments and three replications. Three treatments of Tafethion@ 0.75, 1.0 and 1.25 per cent were compared with three treatments of standard check of Ethion @ 0.75, 1.0 and 1.25 per cent in both the seasons. Water Sprayed apple trees were kept as control. Spraying was done by high volume power sprayer@10 liter spray solution per tree in dormant season and @ 15 liter spray solution per tree in summer season. Pre-treatment count of the pest population recorded one day before the treatment. Post treatment observations were recorded after $1,3,7,15$ and 30 days post spray for the active populations of San Jose scale (SJS) and European red mite (ERM). The pest counts were recorded using hand lens during morning hours. The observations on SJS were recorded from 12 twigs /tree from different directions. $1 \mathrm{~cm}^{2}$ area (bottom, middle and top) was marked on each twig. The number of crawlers of SJS in dormant season, females and nymphs in summer season were recorded from each twig. In case of ERM 12 twigs/ tree, each twig of $10 \mathrm{~cm}$ long were marked for recording the eggs of ERM in dormant season, while as in summer season 20-leaves/ trees were taken for observation on adult and immature stages of ERM. Bio efficacy and phytotoxicity observations were also recorded after spray in summer season at both the locations. Phytotoxicity data such as leaf injury on tips/surface, yellowing, wilting, necrosis, hyponasty and epinasty were recorded after 3,7,15 and 30 days post spraying using (1-10) scale. Yield was calculated on the basis of A grade apple boxes /tree/ treatment at harvesting time. Similarly the observations on natural enemies were also recorded one day before spraying and 1, 5, 7 and 10 days post treatment for each replication. The percent reduction in SJS and ERM population was calculated using the formula given below:

(No. of insects present after treatment x No. of insects present before control)

Per cent reduction $(\mathrm{P})=100 \times 1$ -

(No. of insects present before treatment $\mathrm{x}$ No. of insects present after control)

\section{Results and Discussion}

Bio efficacy

\section{San Jose Scale (dormant season of 2014 \& 2015)}

The results presented in table 1 reveal that all the acaricide treatments were better than the untreated control. Data recorded revealed that at Wakura pooled mean mortality of 68.21 per cent was recorded in Tafethion@1.25 per cent concentration, followed by 62.29 per cent@1.0 per cent concentration and 55.28 per cent @ 0.75 per cent concentration. Standard check (Ethion) recorded highest cumulative mean mortality of 64.48 per cent @ 1.25 per cent concentration, followed by 58.40 per cent @ 1.0 per cent concentration 
and 49.98 per cent @ 0.75 per cent concentration, respectively. Water sprayed plants (control) recorded the lowest pooled mean mortality of 12.17 per cent. In Zaznah pooled mean mortality of 67.69 per cent was recorded in Tafethion @ 1.25 per cent concentration, followed by 59.08 per cent @ 1.0 per cent concentration and 51.21 per cent (a) 0.75 per cent concentration, respectively. Standard check (Ethion) recorded highest cumulative mean mortality of 66.06 per cent @ 1.25 per cent concentration, followed by 61.06 per cent@1.0 per cent concentration and 53.86 per cent @ 0.75 per cent concentration, respectively. Water sprayed plants (control) recorded the lowest pooled mean mortality of 9.40 per cent. All the treatments were significantly different from untreated control, whereas the treatments of Tafethion@1.25, 1.0 and 0.75 per cent were statistically at par with standard check (Ethion)@ 1.5, 2.0 and 2.5 per cent when tested at $P=0.05$ at 30 days after application of treatment. Among the treatments of Tafethion and standard check (Ethion) @ 1.25 1.0and 0.75 percent were at par with each other when tested at $P=0.05$ at 30 days after application of treatment.

\section{San Jose scale (summer season of 2014 \& 2015)}

Perusal of the data in table 2 revealed that at Wakura highest pooled mean mortality of 77.41per cent was recorded in Tafethion @ 1.25 per cent, followed by 71.17 per cent @ 1.0 per cent and 59.39 per cent @ 0.75 per cent. Standard check (Ethion) recorded highest cumulative mean mortality of 75.40 per cent @1.25 per cent, followed by 71.23 per cent @1.0 per cent and 55.89 per cent @ 0.75 per cent, respectively. Water sprayed plants (control) recorded the lowest cumulative mean mortality of 11.18 per cent. In Zaznah pooled mean mortality of 77.17 per cent was recorded in Tafethion@1.25 per cent, followed by 70.83 per cent @ 1.0 per cent and 60.45 per cent @ 0.75 per cent, respectively. Standard check (Ethion) recorded highest cumulative mean mortality of 76.32 per cent @ 1.25 per cent, followed by 71.58 per cent @ 1.0 per cent and 58.91 per cent @0.75 per cent, respectively. Water sprayed plants (control) recorded the lowest pooled mean mortality of 9.30 per cent. All the treatments were significantly different from untreated control, whereas the treatments of Tafethion @ 0.75, 1.0 and 1.25 per cent were statistically at par with standard check (Ethion) @ 0.75, 1.0 and 1.25 per cent when tested at $P=0.05$ at 30 days after application of treatment.

\section{European red mite (dormant season of 2014 \& 2015)}

The results presented in table 1 reveal that all the acaricide treatments were better than the untreated control. Data recorded revealed that at Wakura pooled mean mortality of 76.52 per cent was recorded in Tafethion @ 1.25 per cent concentration, followed by 71.81 per cent @ 1.0 per cent concentration and 64.60 per cent@ 0.75 per cent concentration. Standard check (Ethion) recorded highest cumulative mean mortality of 77.13 per cent @ 1.25 per cent concentration, followed by 73.14 per cent @ 1.0 per cent concentration and 64.22 per cent @ 0.75 per cent concentration, respectively. Water sprayed plants (control) recorded the lowest pooled mean mortality of 7.91 per cent. In Zaznah pooled mean mortality of 75.71 per cent was recorded in Tafethion @ 1.25 per cent concentration, followed by 71.08 per cent @ 1.0 per cent concentration and 63.61 per cent ( 0.75 per cent concentration, respectively. Standard check (Ethion) recorded highest cumulative mean mortality of 76.21 per cent @ 1.25 per cent concentration, followed by 68.93 per cent @ 1.0 per cent concentration and 59.13 per cent @ 0.75 per cent 
concentration, respectively. Water sprayed plants (control) recorded the lowest pooled mean mortality of 9.40 per cent. All the treatments were significantly different from untreated control, whereas the treatments of Tafethion@1.25, 1.0 and 0.75 per cent were statistically at par with standard check (Ethion)@ 1.5, 2.0 and 2.5 per cent when tested at $P=0.05$ at 30 days after application of treatment. Among the treatments of Tafethion and standard check (Ethion) @ 1.25 1.0 and 0.75 percent were at par with each other when tested at $P=0.05$ at 30 days after application of treatment.

\section{European red mite (summer season of 2014 \& 2015)}

Perusal of the data in table 2 revealed that at Wakura highest pooled mean mortality of 82.74 per cent was recorded in Tafethion@ 1.25 per cent, followed by 76.77 per cent @ 1.0 per cent and 68.93 per cent @ 0.75 per cent. Standard check (Ethion) recorded highest cumulative mean mortality of 79.92 per cent @ 1.25 per cent, followed by 77.27 per cent @1.0 per cent and 69.75 per cent @ 0.75 per cent, respectively. Water sprayed plants (control) recorded the lowest cumulative mean mortality of 14.65 per cent. In Zaznah pooled mean mortality of 82.49 per cent was recorded in Tafethion@1.25 per cent, followed by 75.76 per cent @ 1.0 per cent and 73.06 per cent @ 0.75 per cent, respectively. Standard check (Ethion) recorded highest cumulative mean mortality of 80.72 per cent @1.25 per cent, followed by 78.42 per cent @ 1.0 per cent and 72.38 per cent @ 0.75 per cent, respectively. Water sprayed plants (control) recorded the lowest pooled mean mortality of 9.73 per cent. All the treatments were significantly different from untreated control, whereas the treatments of Tafethion @ 0.75,1.0 and 1.25 per cent were statistically at par with standard check (Ethion) @ 0.75, 1.0 and 1.25 per cent when tested at $P=0.05$ at 30 days after application of treatment.

\section{Phytotoxicity on apple plants}

The test doses of Tafethion were sprayed at three concentrations in summer as $0.75,1.0$ and 1.25 for phytotoxicity study. The phytotoxicity observations were recorded on 3, 7, 15 and 30 days post spray. Observations were recorded as leaf injury, yellowing, wilting, necrosis, hyponasty and epinasty. No adverse effect on apple trees were recorded after spray and there were no phytotoxicity symptoms recorded during the evaluation programme in both the years at two locations of Wakura and Zaznah.

\section{Toxicity to Natural Enemies}

\section{Location Wakura}

Toxicity of Tafethionwas evaluated against three important natural enemiesAmblysius, Stethorus punctum and Coccinellids associated with San Jose scale and European red mite in the Kashmir Valley. Highest cumulative mean mortality of natural enemies was recorded as 36.79 per cent in case of Tafethion@1.25 per cent followed by 26.79 per cent @1.0 per cent and 22.79 per cent @ 0.75 per cent respectively. Standard check (Ethion) recorded highest cumulative mean mortality of 29.92 per cent @ 1.25 per cent followed by 25.43 per cent @ 1.0 per cent and 20.96 per cent @0.75 per cent. Pooled mean mortality of 32.90 per cent of Amblyseius was recorded in Tafethion@ 1.25 per cent followed by 25.96 per cent @ 1.0 per cent and 24.10 per cent @ 0.75 per cent. The pooled mean mortality of 30.52 per cent of Amblyseius was recorded in standard check (Ethion)@ 1.25 per cent followed by 25.96 per cent @1.0 per cent and 23.04 per cent @ 0.75 per cent respectively. Highest pooled mean mortality of 36.07 per cent of Stethorus 
punctum was recorded in Tafethion@1.25 per cent followed by 30.10 per cent @ 1.0 per cent and 24.79 per cent @ 0.75 per cent. The pooled mean mortality of 33.78 per cent of Stethorus punctum was recorded in standard check (Ethion)@ 1.25 per cent followed by 26.06 per cent @ 1.0 per cent and 23.61 per cent @ 0.75 per cent respectively. Perusal of data in table 3 revealed that highest pooled mean mortality of 36.80 per cent of Coccinellids was recorded in Tafethion@ @ 1.25 per cent followed by 29.68 per cent @ $1.0 \%$ and 24.25 per cent @ 0.75 per cent. The pooled mean mortality of 33.16 per cent of Coccinellids was recorded in standard check (Ethion)@ 1.25 per cent followed by 28.61 per cent @ 1.0 per cent and 23.48 per cent @ 0.75 per cent respectively.

\section{Location Zaznah}

Perusal of data in table 3 shows highest cumulative mean mortality of natural enemies was recorded as 35.05 per cent in Tafethion @ 1.25 per cent followed by 27.02 per cent @ 1.0 per cent and 24.19 per cent @ 0.75 per cent respectively. Standard check (Ethion) recorded highest cumulative mean mortality of natural enemies 33.68 per cent @ 1.25 per cent followed by 29.29 per cent @ 1.0 per cent and 23.83 per cent @ 0.75 per cent. Pooled mean mortality of 32.36 per cent of Amblyseius was recorded in Tafethion@1.25 per cent followed by 24.61 per cent @ 1.0 per cent and 25.05 per cent @ 0.75 per cent. The pooled mean mortality of 31.88 per cent of Amblyseius was recorded in standard check (Ethion) @ 1.25 per cent followed by 28.63 per cent@1.0 per cent and 22.91per cent @ 0.75 per cent respectively. Highest pooled mean mortality of 37.51 per cent of Stethorus punctum was recorded in Tafethion@ 1.25 per cent followed by 27.75 per cent @ 1.0 per cent and 25.91 per cent @ $0.75 \%$. The pooled mean mortality of 35.45 per cent of Stethorus punctum was recorded in standard check (Ethion) @ 1.25 per cent followed by 29.43 per cent @ 1.0 per cent and 23.96 per cent @ 0.75 per cent respectively. Highest pooled mean mortality of 35.28 per cent of Coccinellids was recorded in Tafethion@ 1.25 per cent followed by 28.72 per cent @ 1.0 per cent and 24.62 per cent @ 0.75 per cent. The pooled mean mortality of 33.51 per cent of Coccinellids was recorded in standard check (Ethion)@ 1.25 per cent followed by 29.80 per cent @ 1.0 per cent and 24.61 per cent @0.75 per cent respectively.

\section{Impact on Yield}

\section{Location Wakura}

The yield in Tafethiontreated trees were recorded as 5.88, 4.63 and 4.30 boxes of $\mathrm{A}$ grade apple/tree for the three different concentrations respectively which was statistically at par with that of standard check (Ethion) treated trees where yield of A grade apple/tree were $5.52,4.74$ and 4.33 boxes in three different concentrations respectively. However, water spray (control) trees recorded the lowest yield of 3.29 boxes of A grade apple/tree as shown in tables 1-4.

\section{Location Zaznah}

The yield in Tafethion treated trees were recorded as 5.58, 4.57 and 4.45 boxes of $\mathrm{A}$ grade apple/tree for the three concentrations respectively which was statistically at par with that of standard check (Ethion) treated trees where yield of A grade apple/tree were $5.83,4.65$ and 4.51 boxes in three different concentrations respectively. However, water spray (control) trees recorded the lowest yield of 3.18 boxes of A grade apple/tree as shown in tables 1-4. The results revealed (pool data of the locations and years) significant differences in mean percent mortality of the target pests of apple in Kashmir due to Tafethion and standard check (Ethion) under trial at different concentrations as compared to the control. 
Table.1 Bioefficacy of Tafethion as dormant spray against San Jose scale Quadraspidiotus perniciosus on apple cv.Red Delicious at Wakura and Zaznah (District Ganderbal) Kashmir (J\&K) during 2014 and 2015

\begin{tabular}{|c|c|c|c|c|c|c|c|c|c|}
\hline \multicolumn{10}{|c|}{ Wakura } \\
\hline \multirow[t]{2}{*}{ Treatment } & \multirow[t]{2}{*}{$\begin{array}{c}\text { Conc. } \\
\%\end{array}$} & \multirow[t]{2}{*}{$\begin{array}{l}\text { Pre- } \\
\text { treatment } \\
\text { count } / \mathrm{cm}^{2}\end{array}$} & \multicolumn{5}{|c|}{$\begin{array}{c}\text { Per cent corrected mortality of San } \\
\text { Jose Scale/cm } \\
\text { (DAT) }\end{array}$} & \multirow[t]{2}{*}{$\begin{array}{l}\text { Pooled } \\
\text { Mean }\end{array}$} & \multirow[t]{2}{*}{$\begin{array}{l}* \text { Yield }(\mathrm{A} \\
\text { grade apple) }\end{array}$} \\
\hline & & & 1 & 3 & 7 & 15 & 30 & & \\
\hline \multirow[t]{3}{*}{ Tafethion } & 0.75 & 33.77 & 24.66 & 41.93 & 55.48 & 77.02 & 82.21 & 55.28 & 4.30 \\
\hline & 1.0 & 33.54 & 41.44 & 49.25 & 59.28 & 75.12 & 86.35 & 62.29 & 4.63 \\
\hline & 1.25 & 31.83 & 45.41 & 55.85 & 67.75 & 79.92 & 91.99 & 68.21 & 5.88 \\
\hline \multirow[t]{3}{*}{ Ethion(Check) } & 0.75 & 30.87 & 22.37 & 29.11 & 45.68 & 71.64 & 81.12 & 49.98 & 4.33 \\
\hline & 1.0 & 30.04 & 32.18 & 44.72 & 54.46 & 72.91 & 87.74 & 58.40 & 4.74 \\
\hline & 1.25 & 28.62 & 40.68 & 47.40 & 64.10 & 79.25 & 91.00 & 64.48 & 5.52 \\
\hline Control & - & 30.84 & 4.32 & 9.06 & 13.07 & 15.68 & 18.75 & 12.17 & 3.29 \\
\hline C.D $(0.05)$ & & & 1.01 & 0.89 & 0.95 & 1.25 & 1.11 & & \\
\hline \multicolumn{10}{|c|}{ Zaznah } \\
\hline \multirow[t]{3}{*}{ Tafethion } & 0.75 & 26.78 & 14.45 & 31.01 & 48.52 & 70.99 & 81.09 & 51.21 & 4.45 \\
\hline & 1.0 & 31.27 & 33.79 & 44.60 & 58.11 & 73.09 & 85.81 & 59.08 & 4.57 \\
\hline & 1.25 & 31.60 & 46.28 & 54.60 & 65.72 & 80.14 & 91.73 & 67.69 & 5.58 \\
\hline \multirow[t]{3}{*}{ Ethion(Check) } & 0.75 & 32.59 & 23.82 & 39.53 & 52.17 & 72.19 & 81.61 & 53.86 & 4.51 \\
\hline & 1.0 & 32.35 & 38.60 & 47.95 & 55.77 & 74.98 & 88.01 & 61.06 & 4.65 \\
\hline & 1.25 & 30.50 & 44.52 & 49.55 & 65.87 & 80.16 & 90.20 & 66.06 & 5.83 \\
\hline Control & - & 37.50 & 3.31 & 7.43 & 9.78 & 11.11 & 15.36 & 9.40 & 3.18 \\
\hline C.D (0.05) & & & 1.05 & 0.99 & 0.76 & 0.87 & 0.92 & & \\
\hline
\end{tabular}

DAT $=$ Days after treatment

$*=$ Boxes/tree 
Table.2 Bioefficacy of Tafethion as summer spray against San Jose scale Quadraspidiotus perniciosuson apple cv.Red Delicious at Wakura and Zaznah (District Ganderbal) Kashmir (J\&K) during 2014 and 2015

\begin{tabular}{|c|c|c|c|c|c|c|c|c|c|}
\hline \multicolumn{10}{|l|}{ Wakura } \\
\hline \multirow[t]{2}{*}{ Treatment } & \multirow[t]{2}{*}{$\begin{array}{c}\text { Conc. } \\
\%\end{array}$} & \multirow[t]{2}{*}{$\begin{array}{l}\text { Pre- } \\
\text { treatment } \\
\text { count } / \mathrm{cm}^{2}\end{array}$} & \multicolumn{5}{|c|}{$\begin{array}{c}\text { Per cent corrected mortality of San } \\
\text { Jose Scale/cm }{ }^{2} \\
\text { (DAT) }\end{array}$} & \multirow[t]{2}{*}{$\begin{array}{l}\text { Pooled } \\
\text { Mean }\end{array}$} & \multirow[t]{2}{*}{$\begin{array}{l}* \text { Yield }(\mathrm{A} \\
\text { grade apple) }\end{array}$} \\
\hline & & & 1 & 3 & 7 & 15 & 30 & & \\
\hline \multirow[t]{3}{*}{ Tafethion } & 0.75 & 8.87 & 38.02 & 46.06 & 63.81 & 76.81 & 84.26 & 59.39 & 4.30 \\
\hline & 1.0 & 10.02 & 47.07 & 61.95 & 75.60 & 83.88 & 87.33 & 71.17 & 4.63 \\
\hline & 1.25 & 9.84 & 61.84 & 68.62 & 81.50 & 85.08 & 90.00 & 77.41 & 5.88 \\
\hline \multirow[t]{3}{*}{ Ethion(Check) } & 0.75 & 8.25 & 36.70 & 42.52 & 59.53 & 73.63 & 82.10 & 55.89 & 4.33 \\
\hline & 1.0 & 8.62 & 47.22 & 61.95 & 76.47 & 81.98 & 88.56 & 71.23 & 4.74 \\
\hline & 1.25 & 10.25 & 57.27 & 67.24 & 78.46 & 84.66 & 89.35 & 75.40 & 5.52 \\
\hline Control & - & 9.50 & 6.10 & 8.01 & 10.30 & 13.88 & 17.66 & 11.18 & 3.29 \\
\hline C.D (0.05) & & & 1.12 & 1.01 & 0.85 & 0.96 & 0.79 & & \\
\hline \multicolumn{10}{|c|}{ Zaznah } \\
\hline \multirow[t]{3}{*}{ Tafethion } & 0.75 & 8.70 & 24.39 & 49.84 & 67.05 & 76.80 & 84.16 & 60.45 & 4.45 \\
\hline & 1.0 & 9.59 & 51.03 & 58.71 & 73.80 & 83.77 & 86.85 & 70.83 & 4.57 \\
\hline & 1.25 & 9.70 & 61.71 & 67.05 & 80.77 & 85.26 & 91.08 & 77.17 & 5.58 \\
\hline \multirow[t]{3}{*}{ Ethion(Check) } & 0.75 & 8.69 & 27.92 & 47.44 & 61.94 & 74.90 & 82.35 & 58.91 & 4.51 \\
\hline & 1.0 & 10.17 & 49.51 & 62.67 & 74.24 & 83.25 & 88.22 & 71.58 & 4.65 \\
\hline & 1.25 & 9.34 & 61.15 & 67.31 & 79.17 & 84.05 & 89.90 & 76.32 & 5.83 \\
\hline Control & - & 9.58 & 5.03 & 6.77 & 8.32 & 11.08 & 15.29 & 9.30 & 3.18 \\
\hline C.D (0.05) & & & 1.00 & 1.25 & 1.34 & 1.14 & 1.87 & & \\
\hline
\end{tabular}

DAT $=$ Days after treatment

$*=$ Boxes/tree 
Table.3 Bio efficacy of Tafethion as dormant spray against European red mite Panonychus ulmi on apple cv. Red Delicious at Wakura and Zaznah (District Ganderbal) Kashmir (J\&K) during 2014 and 2015

\begin{tabular}{|c|c|c|c|c|c|c|c|c|c|}
\hline \multicolumn{10}{|c|}{ Wakura } \\
\hline \multirow[t]{2}{*}{ Treatment } & \multirow[t]{2}{*}{$\begin{array}{c}\text { Conc. } \\
\%\end{array}$} & \multirow{2}{*}{$\begin{array}{l}\text { Pre- } \\
\text { treatment } \\
\text { count per } \\
\text { twig } \\
\end{array}$} & \multicolumn{5}{|c|}{$\begin{array}{l}\text { Percent mortality of European Red } \\
\text { Mite/twig } \\
\text { (DAT) }\end{array}$} & \multirow[t]{2}{*}{$\begin{array}{l}\text { Pooled } \\
\text { Mean }\end{array}$} & \multirow[t]{2}{*}{$\begin{array}{l}* \text { Yield }(\mathrm{A} \\
\text { grade apple }\end{array}$} \\
\hline & & & 1 & 3 & 7 & 15 & 30 & & \\
\hline \multirow[t]{3}{*}{ Tafethion } & 0.75 & 44.50 & 30.84 & 47.02 & 67.90 & 85.84 & 91.39 & 64.60 & 4.30 \\
\hline & 1.0 & 41.72 & 41.24 & 59.86 & 74.34 & 89.05 & 94.57 & 71.81 & 4.63 \\
\hline & 1.25 & 41.62 & 48.08 & 65.06 & 80.90 & 91.71 & 96.87 & 76.52 & 5.88 \\
\hline \multirow{3}{*}{ Ethion(Check) } & 0.75 & 43.72 & 29.92 & 46.79 & 67.71 & 85.57 & 91.13 & 64.22 & 4.33 \\
\hline & 1.0 & 43.80 & 44.04 & 61.84 & 75.39 & 89.71 & 94.70 & 73.14 & 4.74 \\
\hline & 1.25 & 42.59 & 49.41 & 66.01 & 81.62 & 91.71 & 96.87 & 77.13 & 5.52 \\
\hline Control & - & 44.33 & 3.86 & 6.08 & 8.17 & 9.07 & 12.39 & 7.91 & 3.29 \\
\hline C.D (0.05) & & & 0.84 & 0.76 & 0.99 & 1.05 & 1.14 & & \\
\hline \multicolumn{10}{|c|}{ Zaznah } \\
\hline \multirow[t]{3}{*}{ Tafethion } & 0.75 & 39.62 & 29.11 & 48.82 & 66.67 & 83.93 & 89.53 & 63.61 & 4.45 \\
\hline & 1.0 & 38.42 & 45.50 & 58.17 & 71.09 & 87.37 & 93.30 & 71.08 & 4.57 \\
\hline & 1.25 & 37.05 & 50.84 & 63.40 & 78.21 & 90.05 & 96.07 & 75.71 & 5.58 \\
\hline \multirow[t]{3}{*}{ Ethion(Check) } & 0.75 & 37.30 & 26.55 & 37.97 & 59.46 & 82.60 & 89.06 & 59.13 & 4.51 \\
\hline & 1.0 & 37.98 & 40.19 & 55.63 & 69.20 & 85.85 & 93.76 & 68.93 & 4.65 \\
\hline & 1.25 & 38.40 & 49.97 & 65.31 & 78.62 & 90.84 & 96.29 & 76.21 & 5.83 \\
\hline Control & - & 37.50 & 3.46 & 6.88 & 10.05 & 11.34 & 15.23 & 9.40 & 3.18 \\
\hline C.D (0.05) & & & 0.94 & 1.16 & 1.20 & 0.97 & 0.87 & & \\
\hline
\end{tabular}

DAT=Days after treatment $*=$ Boxes/tree. 
Table.4 Bio efficacy of Tafethion as summer spray against European red mite Panonychus ulmi on apple cv. Red Delicious at Wakura and Zaznah (District Ganderbal) Kashmir (J\&K) during 2014 and 2015

\begin{tabular}{|c|c|c|c|c|c|c|c|c|c|}
\hline \multicolumn{10}{|c|}{ Wakura } \\
\hline \multirow[t]{2}{*}{ Treatment } & \multirow[t]{2}{*}{$\begin{array}{c}\text { Conc. } \\
\%\end{array}$} & \multirow{2}{*}{\begin{tabular}{|l|} 
Pre- \\
treatment \\
count per \\
leaf
\end{tabular}} & \multicolumn{5}{|c|}{$\begin{array}{c}\text { Per cent corrected mortality of } \\
\text { European red mite per leaf } \\
\text { (DAT) }\end{array}$} & \multirow[t]{2}{*}{$\begin{array}{l}\text { Pooled } \\
\text { Mean }\end{array}$} & \multirow[t]{2}{*}{$\begin{array}{l}* \text { Yield }(\mathrm{A} \\
\text { grade apple) }\end{array}$} \\
\hline & & & 1 & 3 & 7 & 15 & 30 & & \\
\hline \multirow[t]{3}{*}{ Tafethion } & 0.75 & 8.82 & 32.92 & 63.56 & 76.66 & 84.59 & 86.95 & 68.93 & 4.30 \\
\hline & 1.0 & 9.40 & 54.90 & 70.23 & 81.95 & 87.46 & \begin{tabular}{|l|}
89.30 \\
\end{tabular} & 76.77 & 4.63 \\
\hline & 1.25 & 9.74 & 68.45 & 76.98 & 86.09 & 89.70 & 92.46 & 82.74 & 5.88 \\
\hline \multirow[t]{3}{*}{ Ethion(Check) } & 0.75 & 8.94 & 36.61 & 65.06 & 76.42 & 84.17 & 86.49 & 69.75 & 4.33 \\
\hline & 1.0 & 8.97 & 57.27 & 70.43 & 81.40 & 87.44 & 89.83 & 77.27 & 4.74 \\
\hline & 1.25 & 9.65 & 61.92 & 75.03 & 83.82 & 88.56 & 90.29 & 79.92 & 5.52 \\
\hline Control & - & 9.50 & 6.83 & 10.98 & 15.43 & 18.53 & 21.43 & 14.65 & 3.29 \\
\hline C.D $(0.05)$ & & & 0.76 & 1.01 & 1.25 & 0.85 & 0.74 & & \\
\hline \multicolumn{10}{|c|}{ Zaznah } \\
\hline \multirow[t]{3}{*}{ Tafethion } & 0.75 & 10.04 & 51.45 & 70.60 & 74.88 & 80.49 & 87.86 & 73.06 & 4.45 \\
\hline & 1.0 & 10.04 & 52.69 & 74.78 & 78.14 & 84.37 & 88.81 & 75.76 & 4.57 \\
\hline & 1.25 & 9.84 & 68.11 & 81.51 & 84.38 & 87.45 & 91.00 & 82.49 & 5.58 \\
\hline \multirow[t]{3}{*}{ Ethion(Check) } & 0.75 & 9.50 & 46.06 & 69.66 & 76.83 & 82.21 & 87.17 & 72.38 & 4.51 \\
\hline & 1.0 & 10.07 & 55.78 & 77.85 & 82.15 & 86.33 & 90.00 & 78.42 & 4.65 \\
\hline & 1.25 & 8.42 & 63.48 & 78.53 & 83.45 & 87.45 & 90.70 & 80.72 & 5.83 \\
\hline Control & - & 9.43 & 5.27 & 7.38 & 8.47 & 12.03 & 15.54 & 9.73 & 3.18 \\
\hline C.D (0.05) & & & 0.79 & 0.99 & 0.89 & 1.20 & 1.01 & & \\
\hline
\end{tabular}

DAT= Days after treatment

$*=$ Boxes/tree. 
Table.5 Toxicity of Tafethion as summer spray against natural enemies of San Jose Scale and European red mite on apple cv. Red Delicious at Wakura and Zaznah, (District Ganderbal) Kashmir (J\&K) during 2014 and 2015

\begin{tabular}{|c|c|c|c|c|c|c|c|c|c|c|c|c|c|c|c|c|c|c|c|c|}
\hline \multirow{3}{*}{ Treatments } & \multirow{3}{*}{ Conc. \% } & \multicolumn{3}{|c|}{$\begin{array}{l}\text { Pre-treatment } \\
\text { count }\end{array}$} & \multicolumn{12}{|c|}{$\begin{array}{l}\text { Post treatment count* } \\
\text { (Mean population of Natural Enemies (DAT) }\end{array}$} & \multicolumn{3}{|c|}{$\begin{array}{l}\text { Pooled mean \% } \\
\text { mortality of NE }\end{array}$} & \multirow{3}{*}{$\begin{array}{c}\text { Cumulative } \\
\text { Mean \% } \\
\text { mortality o } \\
\text { NE }\end{array}$} \\
\hline & & \multirow[t]{2}{*}{$\mathbf{A}$} & \multirow[t]{2}{*}{$\mathbf{S}$} & \multirow[t]{2}{*}{$\mathbf{C}$} & \multicolumn{4}{|c|}{$\mathbf{A}$} & \multicolumn{4}{|c|}{$\mathbf{S}$} & \multicolumn{4}{|c|}{$\mathrm{C}$} & \multirow[b]{2}{*}{$\mathbf{A}$} & \multirow[b]{2}{*}{$\mathbf{S}$} & \multirow[b]{2}{*}{$\mathrm{C}$} & \\
\hline & & & & & 1 & 5 & 7 & 10 & 1 & 5 & 7 & 10 & 1 & 5 & 7 & 10 & & & & \\
\hline & & \multicolumn{19}{|c|}{ Wakura } \\
\hline \multirow[t]{3}{*}{ Tafethion } & 0.75 & 1.82 & 1.45 & 1.00 & $\begin{array}{c}1.59 \\
(12.39)\end{array}$ & $\begin{array}{c}1.41 \\
(22.31)\end{array}$ & $\begin{array}{c}1.30 \\
(28.37)\end{array}$ & $\begin{array}{c}1.21 \\
(33.33)\end{array}$ & $\begin{array}{l}1.34 \\
(8.1)\end{array}$ & $\begin{array}{c}1.17 \\
(18.74)\end{array}$ & $\begin{array}{c}0.99 \\
(31.20)\end{array}$ & $\begin{array}{c}0.84 \\
(41.08)\end{array}$ & $\begin{array}{c}0.88 \\
(12.00)\end{array}$ & $\begin{array}{c}0.80 \\
(20.00)\end{array}$ & $\begin{array}{c}0.70 \\
(30.00)\end{array}$ & $\begin{array}{c}0.65 \\
(35.00)\end{array}$ & $\begin{array}{c}1.38 \\
(24.10)\end{array}$ & $\begin{array}{c}1.09 \\
(24.79)\end{array}$ & $\begin{array}{c}0.76 \\
(24.25)\end{array}$ & 22.79 \\
\hline & 1.0 & 1.82 & 1.00 & 0.99 & $\begin{array}{c}1.50 \\
(17.58)\end{array}$ & $\begin{array}{c}1.41 \\
(22.53)\end{array}$ & $\begin{array}{c}1.30 \\
(28.57)\end{array}$ & $\begin{array}{c}1.18 \\
(35.16) \\
\end{array}$ & $\begin{array}{c}0.84 \\
(15.52)\end{array}$ & $\begin{array}{c}0.74 \\
(25.58)\end{array}$ & $\begin{array}{c}0.63 \\
(36.64)\end{array}$ & $\begin{array}{c}0.57 \\
(42.68)\end{array}$ & $\begin{array}{c}0.87 \\
(11.65)\end{array}$ & $\begin{array}{c}0.74 \\
(24.85)\end{array}$ & $\begin{array}{c}0.62 \\
(37.04)\end{array}$ & $\begin{array}{c}0.54 \\
(45.16)\end{array}$ & $\begin{array}{c}1.35 \\
(25.96)\end{array}$ & $\begin{array}{c}0.70 \\
(30.10)\end{array}$ & $\begin{array}{c}0.69 \\
(29.68)\end{array}$ & 26.79 \\
\hline & 1.25 & 1.76 & 0.98 & 1.01 & $\begin{array}{c}1.40 \\
(20.18)\end{array}$ & $\begin{array}{c}1.23 \\
(30.15)\end{array}$ & $\begin{array}{c}1.36 \\
(37.96)\end{array}$ & $\begin{array}{c}1.00 \\
(43.30) \\
\end{array}$ & $\begin{array}{c}0.84 \\
(13.73)\end{array}$ & $\begin{array}{c}0.69 \\
(29.14)\end{array}$ & $\begin{array}{c}0.52 \\
(46.59) \\
\end{array}$ & $\begin{array}{c}0.44 \\
(54.81)\end{array}$ & $\begin{array}{c}0.84 \\
(16.40)\end{array}$ & $\begin{array}{c}0.69 \\
(31.32) \\
\end{array}$ & $\begin{array}{c}0.54 \\
(46.25)\end{array}$ & $\begin{array}{c}0.47 \\
(53.22) \\
\end{array}$ & $\begin{array}{c}1.18 \\
(32.90)\end{array}$ & $\begin{array}{c}0.62 \\
(36.07) \\
\end{array}$ & $\begin{array}{c}0.64 \\
(36.80)\end{array}$ & 36.79 \\
\hline \multirow[t]{3}{*}{$\begin{array}{l}\text { Ethion } \\
\text { (Check) }\end{array}$} & 0.75 & 1.79 & 1.08 & 0.99 & $\begin{array}{c}1.59 \\
(11.17) \\
\end{array}$ & $\begin{array}{c}1.41 \\
(21.23) \\
\end{array}$ & $\begin{array}{c}1.30 \\
(27.37) \\
\end{array}$ & $\begin{array}{c}1.21 \\
(32.40) \\
\end{array}$ & $\begin{array}{c}0.97 \\
(10.19) \\
\end{array}$ & $\begin{array}{c}0.89 \\
(17.59) \\
\end{array}$ & $\begin{array}{c}0.77 \\
(28.70) \\
\end{array}$ & $\begin{array}{c}0.67 \\
(37.96) \\
\end{array}$ & $\begin{array}{c}0.88 \\
(11.11) \\
\end{array}$ & $\begin{array}{c}0.80 \\
(19.19) \\
\end{array}$ & $\begin{array}{c}0.70 \\
(29.29) \\
\end{array}$ & $\begin{array}{c}0.65 \\
(34.34) \\
\end{array}$ & $\begin{array}{c}1.38 \\
(23.04)\end{array}$ & $\begin{array}{c}0.83 \\
(23.61) \\
\end{array}$ & $\begin{array}{c}0.76 \\
(23.48)\end{array}$ & 20.96 \\
\hline & 1.0 & 1.82 & 0.94 & 0.97 & $\begin{array}{c}1.50 \\
(17.58) \\
\end{array}$ & $\begin{array}{c}1.41 \\
(22.53) \\
\end{array}$ & $\begin{array}{c}1.30 \\
(28.57) \\
\end{array}$ & $\begin{array}{c}1.18 \\
(35.16) \\
\end{array}$ & $\begin{array}{c}0.84 \\
(10.64) \\
\end{array}$ & $\begin{array}{c}0.74 \\
(21.28) \\
\end{array}$ & $\begin{array}{c}0.63 \\
(32.98) \\
\end{array}$ & $\begin{array}{c}0.57 \\
(39.36) \\
\end{array}$ & $\begin{array}{c}0.87 \\
(10.31) \\
\end{array}$ & $\begin{array}{c}0.74 \\
(23.71) \\
\end{array}$ & $\begin{array}{c}0.62 \\
(36.08) \\
\end{array}$ & $\begin{array}{c}0.54 \\
(44.33) \\
\end{array}$ & $\begin{array}{c}1.35 \\
(25.96) \\
\end{array}$ & $\begin{array}{c}0.70 \\
(26.06) \\
\end{array}$ & $\begin{array}{c}0.69 \\
(28.61) \\
\end{array}$ & 25.43 \\
\hline & 1.25 & 1.72 & 0.94 & 0.95 & $\begin{array}{c}1.39 \\
(19.19) \\
\end{array}$ & $\begin{array}{c}1.21 \\
(29.65) \\
\end{array}$ & $\begin{array}{c}1.44 \\
(31.98) \\
\end{array}$ & $\begin{array}{c}1.01 \\
(41.28) \\
\end{array}$ & $\begin{array}{c}0.84 \\
(10.64) \\
\end{array}$ & $\begin{array}{c}0.69 \\
(26.60) \\
\end{array}$ & $\begin{array}{c}0.52 \\
(44.68) \\
\end{array}$ & $\begin{array}{c}0.44 \\
(53.19) \\
\end{array}$ & $\begin{array}{c}0.86 \\
(11.58) \\
\end{array}$ & $\begin{array}{c}0.69 \\
(27.37) \\
\end{array}$ & $\begin{array}{c}0.54 \\
(43.16) \\
\end{array}$ & $\begin{array}{c}0.47 \\
(50.53) \\
\end{array}$ & $\begin{array}{c}1.20 \\
(30.52) \\
\end{array}$ & $\begin{array}{c}0.62 \\
(33.78) \\
\end{array}$ & $\begin{array}{c}0.64 \\
(33.16) \\
\end{array}$ & 29.92 \\
\hline $\begin{array}{c}\text { Water } \\
\text { (Control) }\end{array}$ & - & 1.77 & 0.97 & 0.95 & $\begin{array}{c}1.70 \\
(3.95)\end{array}$ & $\begin{array}{c}1.67 \\
(5.65)\end{array}$ & $\begin{array}{c}1.64 \\
(7.34)\end{array}$ & $\begin{array}{l}1.60 \\
(9.6)\end{array}$ & $\begin{array}{c}0.95 \\
(2.06)\end{array}$ & $\begin{array}{c}0.93 \\
(4.12)\end{array}$ & $\begin{array}{c}0.90 \\
(7.22)\end{array}$ & $\begin{array}{c}0.90 \\
(7.22)\end{array}$ & $\begin{array}{c}0.92 \\
(3.16)\end{array}$ & $\begin{array}{c}0.90 \\
(5.26)\end{array}$ & $\begin{array}{c}0.86 \\
(9.47)\end{array}$ & $\begin{array}{c}0.84 \\
(15.43)\end{array}$ & $\begin{array}{c}1.63 \\
(6.64)\end{array}$ & $\begin{array}{c}0.91 \\
(5.15)\end{array}$ & $\begin{array}{c}0.84 \\
(8.32)\end{array}$ & 6.70 \\
\hline \multicolumn{2}{|l|}{ C.D $(\mathbf{P} \leq 0.05)$} & & & & 1.31 & 0.58 & 1.05 & 1.14 & 1.06 & 1.13 & 1.05 & 1.46 & 0.88 & 0.78 & 0.35 & 0.74 & & & & \\
\hline & \multicolumn{20}{|c|}{ Zaznah } \\
\hline \multirow[t]{3}{*}{ Tafethion } & 0.75 & 1.77 & 1.10 & 1.00 & $\begin{array}{c}1.58 \\
(10.73)\end{array}$ & $\begin{array}{c}1.42 \\
(19.77)\end{array}$ & $\begin{array}{c}1.30 \\
(26.30)\end{array}$ & $\begin{array}{c}1.21 \\
(31.40)\end{array}$ & $\begin{array}{c}0.97 \\
(12.28)\end{array}$ & $\begin{array}{c}0.88 \\
(20.46)\end{array}$ & $\begin{array}{c}0.76 \\
(30.91)\end{array}$ & $\begin{array}{c}0.66 \\
(40.00)\end{array}$ & $\begin{array}{c}0.87 \\
(13.00)\end{array}$ & $\begin{array}{c}0.80 \\
(20.50)\end{array}$ & $\begin{array}{c}0.70 \\
(29.80)\end{array}$ & $\begin{array}{c}0.65 \\
(34.99)\end{array}$ & $\begin{array}{c}1.38 \\
(25.05)\end{array}$ & $\begin{array}{c}0.82 \\
(25.91)\end{array}$ & $\begin{array}{c}0.76 \\
(24.62)\end{array}$ & 24.19 \\
\hline & 1.0 & 1.79 & 0.96 & 0.98 & $\begin{array}{c}1.64 \\
(8.43) \\
\end{array}$ & $\begin{array}{c}1.36 \\
(23.54)\end{array}$ & $\begin{array}{c}1.26 \\
(29.64) \\
\end{array}$ & $\begin{array}{c}1.14 \\
(36.33)\end{array}$ & $\begin{array}{c}0.83 \\
(13.08) \\
\end{array}$ & $\begin{array}{c}0.73 \\
(23.56) \\
\end{array}$ & $\begin{array}{c}0.63 \\
(34.03) \\
\end{array}$ & $\begin{array}{c}0.57 \\
(40.32) \\
\end{array}$ & $\begin{array}{c}0.88 \\
(9.75) \\
\end{array}$ & $\begin{array}{c}0.73 \\
(25.12) \\
\end{array}$ & $\begin{array}{c}0.62 \\
(36.41) \\
\end{array}$ & $\begin{array}{c}0.55 \\
(43.60) \\
\end{array}$ & $\begin{array}{c}1.35 \\
(24.61) \\
\end{array}$ & $\begin{array}{c}0.70 \\
(27.75) \\
\end{array}$ & $\begin{array}{c}0.70 \\
(28.72) \\
\end{array}$ & 27.02 \\
\hline & 1.25 & 1.72 & 0.99 & 0.99 & $\begin{array}{c}1.34 \\
(22.16) \\
\end{array}$ & $\begin{array}{c}1.19 \\
(30.90)\end{array}$ & $\begin{array}{c}1.14 \\
(33.82) \\
\end{array}$ & $\begin{array}{c}0.99 \\
(42.57) \\
\end{array}$ & $\begin{array}{c}0.83 \\
(15.67)\end{array}$ & $\begin{array}{c}0.68 \\
(30.91) \\
\end{array}$ & $\begin{array}{c}0.52 \\
(47.14) \\
\end{array}$ & $\begin{array}{c}0.43 \\
(56.33) \\
\end{array}$ & $\begin{array}{c}0.86 \\
(12.68) \\
\end{array}$ & $\begin{array}{c}0.68 \\
(30.97)\end{array}$ & $\begin{array}{c}0.54 \\
(45.18) \\
\end{array}$ & $\begin{array}{c}0.47 \\
(51.53) \\
\end{array}$ & $\begin{array}{c}1.16 \\
(32.36) \\
\end{array}$ & $\begin{array}{c}0.62 \\
(37.51)\end{array}$ & $\begin{array}{c}0.64 \\
(35.28) \\
\end{array}$ & 35.05 \\
\hline \multirow[t]{3}{*}{$\begin{array}{l}\text { Ethion } \\
\text { (Check) }\end{array}$} & 0.75 & 1.79 & 1.09 & 1.01 & $\begin{array}{c}1.60 \\
(10.89) \\
\end{array}$ & $\begin{array}{c}1.42 \\
(20.95) \\
\end{array}$ & $\begin{array}{c}1.30 \\
(27.37) \\
\end{array}$ & $\begin{array}{c}1.21 \\
(32.40) \\
\end{array}$ & $\begin{array}{c}0.97 \\
(10.60) \\
\end{array}$ & $\begin{array}{c}0.89 \\
(17.97) \\
\end{array}$ & $\begin{array}{c}0.77 \\
(29.03) \\
\end{array}$ & $\begin{array}{c}0.67 \\
(38.25) \\
\end{array}$ & $\begin{array}{c}0.88 \\
(12.42) \\
\end{array}$ & $\begin{array}{c}0.80 \\
(20.38) \\
\end{array}$ & $\begin{array}{c}0.70 \\
(30.33) \\
\end{array}$ & $\begin{array}{c}0.65 \\
(35.31)\end{array}$ & $\begin{array}{c}1.38 \\
(22.91)\end{array}$ & $\begin{array}{c}0.83 \\
(23.96)\end{array}$ & $\begin{array}{c}0.76 \\
(24.61)\end{array}$ & 23.83 \\
\hline & 1.0 & 1.79 & 1.37 & 1.37 & $\begin{array}{c}1.55 \\
(13.08)\end{array}$ & $\begin{array}{c}1.34 \\
(25.27)\end{array}$ & $\begin{array}{c}1.20 \\
(32.86)\end{array}$ & $\begin{array}{c}1.02 \\
(43.30)\end{array}$ & $\begin{array}{c}1.22 \\
(10.85)\end{array}$ & $\begin{array}{c}1.02 \\
(24.54)\end{array}$ & $\begin{array}{c}0.87 \\
(35.94)\end{array}$ & $\begin{array}{c}0.71 \\
(46.41)\end{array}$ & $\begin{array}{c}1.24 \\
(10.16)\end{array}$ & $\begin{array}{c}1.00 \\
(26.45)\end{array}$ & $\begin{array}{c}0.87 \\
(36.55)\end{array}$ & $\begin{array}{c}0.74 \\
(46.03)\end{array}$ & $\begin{array}{c}1.28 \\
(28.63)\end{array}$ & $\begin{array}{c}0.96 \\
(29.43)\end{array}$ & $\begin{array}{c}0.96 \\
(29.80)\end{array}$ & 29.29 \\
\hline & 1.25 & 1.76 & 0.97 & 0.96 & $\begin{array}{c}1.39 \\
(20.99)\end{array}$ & $\begin{array}{c}1.21 \\
(31.22)\end{array}$ & $\begin{array}{c}1.17 \\
(33.49)\end{array}$ & $\begin{array}{c}1.01 \\
(42.59)\end{array}$ & $\begin{array}{c}0.84 \\
(12.90)\end{array}$ & $\begin{array}{c}0.69 \\
(28.45)\end{array}$ & $\begin{array}{c}0.52 \\
(46.08)\end{array}$ & $\begin{array}{c}0.44 \\
(54.38)\end{array}$ & $\begin{array}{c}0.84 \\
(12.04)\end{array}$ & $\begin{array}{c}0.69 \\
(27.75)\end{array}$ & $\begin{array}{c}0.54 \\
(43.46)\end{array}$ & $\begin{array}{c}0.47 \\
(50.79)\end{array}$ & $\begin{array}{c}1.20 \\
(31.88)\end{array}$ & $\begin{array}{c}0.62 \\
(35.45)\end{array}$ & $\begin{array}{c}0.64 \\
(33.51)\end{array}$ & 33.68 \\
\hline $\begin{array}{c}\text { Water } \\
\text { (Control) }\end{array}$ & - & 1.77 & 1.39 & 0.97 & $\begin{array}{c}1.71 \\
(3.95) \\
\end{array}$ & $\begin{array}{c}1.67 \\
(5.38) \\
\end{array}$ & $\begin{array}{c}1.64 \\
(7.08)\end{array}$ & $\begin{array}{c}1.61 \\
(9.06) \\
\end{array}$ & $\begin{array}{c}1.33 \\
(4.04) \\
\end{array}$ & $\begin{array}{c}1.24 \\
(9.25)\end{array}$ & $\begin{array}{c}1.11 \\
(16.86) \\
\end{array}$ & $\begin{array}{c}1.05 \\
(20.41) \\
\end{array}$ & $\begin{array}{c}0.93 \\
(4.11) \\
\end{array}$ & $\begin{array}{c}0.90 \\
(7.68) \\
\end{array}$ & $\begin{array}{c}0.87 \\
(10.29) \\
\end{array}$ & $\begin{array}{c}0.85 \\
(16.72) \\
\end{array}$ & $\begin{array}{c}1.66 \\
(6.23) \\
\end{array}$ & $\begin{array}{c}1.18 \\
(12.64) \\
\end{array}$ & $\begin{array}{c}0.89 \\
(9.66)\end{array}$ & $\begin{array}{l}9.5 \\
1 \\
\end{array}$ \\
\hline \multicolumn{2}{|l|}{ C.D(P $\leq 0.05)$} & & & & 1.05 & 0.68 & 0.75 & 0.57 & 1.03 & 1.00 & 1.04 & 1.08 & 1.29 & 1.47 & 0.68 & 0.78 & & & & \\
\hline
\end{tabular}

*Mean of 3 replicates, Figures in Parenthesis are \% Mortality of Natural enemies; $\mathrm{A}=$ Amblysius, $\mathrm{S}=$ Stethorus punctum, $\mathrm{C}=$ Coccinellids, N.E= Natural enemies

The population of $\mathrm{A}$ and $\mathrm{S}$ recorded on the basis of per 6 leaves and $\mathrm{C}$ on the basis of 4 branches. 
The pooled data (2014 and 2015) indicated significant reduction in SJS and ERM populations at 1.0 per cent and 0.75 per cent in dormant and summer. It is concluded from the present investigation that Tafethion @ 1.25 per cent recorded highest mean mortality 68.21 per cent against SJS and Ethion @ 1.25 $\%$ recorded 77.13 per cent against ERM as dormant spray at Wakura location and that of 77.41 per cent and 82.49 per cent pooled mean mortality@1.25 per cent of SJS and ERM as summer spray at Wakura and Zaznah location respectively. The maximum yield of (5.88 boxes/tree) of A grade apple was found in Tafethion sprayed @ 1.25 per cent at Wakura location. None of the phytotoxicity effects were recorded on apple leaves during 2014 and 2015. Maximum pooled mortality of natural enemies was recorded as 36.79 per cent @ 1.25 per cent against the Tafethion at Wakura location. The studies are more or less in conformity with the studies of Lal (1952), and Hix et al., (1999) who reported the similar results. Khajuria and Sharma (1999) reported that San Jose scale infestation was significantly reduced by the application of 2.0 per cent miscible oil. These results are also in agreement with that of Sahuet al., 2008. They reported Dicofol as the most toxic chemical to tea red spider mite, Amblyseius longispinosus with an LC50 of 0.0156 per cent. Bhanuet al., (2006) has reported Dicofol and Ethion resulted in the lowest number of sheath mite (10.3 and 11.3/10 spikelets) in rice. As conclusion, Tafethion is found very effective against pest's viz., San Jose scale and European red mite in apple orchards with a unique mode of action, and thus a potential acaricide in integrated management of SJS and ERM on Apple.

\section{References}

Abbot, W.S. 1925. A method of computing the effectiveness of insecticides. $J$. Economic Entomol., 18: 265-267.
Anonymous. 2014. Management of pests on apple in Kashmir, Division of Entomology, Directorate of Extension education, SKUAST-K, Shalimar.

Bhalla, O.P. and Gupta, P.R. 1993. Insect pest of temperate fruits. Advances in Horticulture - Fruit Crops Malhotra Publishing House, New Delhi, India, 3: 1557-1589.

Bhanu, K.V., Reddy, P.S. and Zeheruddeen, S.M. 2006. Evaluation of some acaricide against leaf mite and sheath mite in rice. Indian J. Plant Protection, 34(1): 132-133

Bhardwaj, S.P. 1988. Effect of summer applications of insecticides on San Jose scale (Quadraspidiotus perniciosus) in orchards of apple (Malus pirmila). Indian J. Agri. Sci., 58: 655-656.

Chapman, P.J. 1967. Petroleum oils for the control of orchard pests. N.Y. Agric. Exp. Stn. Bull., 814.

Sahu, B., Chakraborty, G., and Somachoudhary, A.K. 2008. Efficacy of Spiromesifen $240 \mathrm{SC}$ in the management of tea red spider mite (Oligoonychuscoffee Niether) on tea. Environ. Ecol., 24(1) retrieved from http://d.wanfangdata.com.cn/NSTLQK NSTL_QK12332409.aspx

Badenes-Perez, F.R., F.G. Zalom \& W.J. Bentley. 2010. Effects of dormant insecticide treatments on the San Jose scale (Homoptera: Diaspididae) and its parasitoids Encarsia perniciosiand Aphytis spp. (Hymenoptera: Aphelinidae). Int. J. Pest Manage., 26.

Hix, R.L., Pless, C.D., Deyton, D.E. and Sams, C.E. (1999) Management of San Jose Scale on apple with soybean oil dormant sprays. Horticulture Sci., 34(1): 106-108.

Khajuria, D.R. and Sharma, H.K. 1999. Use of miscible oil and insecticidal combinations in management of San Jose scale (Quadraspidiotus 
perniciosus) on apple (Malus domestica). Indian J. Agri. Sci., 67: 488-489.

Lal, K.B. 1952. Insect pests of fruit trees grown in the plain of the Uttar Pardesh and their control. Agri. Animal Husbandary, 3(1-2): 54-80.

M.A. Mir, R.K. Nehru, Showkat Ahmad, Shabeenamajid and P. Jalaludin.2015. Efficacy of some horticultural mineral oils (HMO's) against Quadraspidiotus perniciosus (Comstock) in Kashmir, Green Farming, 6(5): 1126-1129.

Madson, H. and Morgan, C.V.G. 1970. Pome fruit pest's ad their control. J. Entomol. Sci., 37: 41-45.

Singh. 1964. Temperate fruit pests in India. Entomol. Soc., 5-261-300.

Singh, S.S., Tiwari. H.C. and Rai, K.M. 2001.Evaluation of some modern insecticides against San Jose scale, Quadraspidiotus perniciosus (Comstock) on apple. J. Entomol. Res., 25: 69-71.

Sud, V.K., Hameed, S.F. and Sharma, L.D. 1975. The insecticidal control of San Jose scale on apple. J. Horticulture, 50(2): 165-168.

\section{How to cite this article:}

Asma Sherwani, Malik Mukhtar Ahmad, Muneer Ahmad Sofi and Saima Maqsood. 2017. Field Evaluation of New Ovicidal Acaricide (Tafethion) against Two Major Insect Pests of Apple in Kashmir. Int.J.Curr.Microbiol.App.Sci. 6(7): 3905-3916. doi: https://doi.org/10.20546/ijcmas.2017.607.403 